\title{
Understanding the Anticontagion Process and Reopening of China during COVID-19 via Coevolution Network of Epidemic and Awareness
}

\author{
Lingbo Li $\mathbb{D}$, Ying Fan $\mathbb{D}^{\mathbb{D}}$, An Zeng $\mathbb{D}$, and Zengru Di \\ School of Systems Science, Beijing Normal University, Beijing 100875, China \\ Correspondence should be addressed to Ying Fan; yfan@bnu.edu.cn and An Zeng; anzeng@bnu.edu.cn
}

Received 14 October 2020; Revised 29 December 2020; Accepted 16 April 2021; Published 11 May 2021

Academic Editor: Ning Cai

Copyright (c) 2021 Lingbo Li et al. This is an open access article distributed under the Creative Commons Attribution License, which permits unrestricted use, distribution, and reproduction in any medium, provided the original work is properly cited.

\begin{abstract}
The novel coronavirus (COVID-19) pandemic is intensifying all over the world, but some countries, including China, have developed extensive and successful experience in controlling this pandemic. In this context, some questions arise naturally: What can countries caught up in the epidemic learn from China's experience? In regions where the outbreak is under control, what would lead to a resurgence of the epidemic? To address these issues, we investigate China's experience in anticontagion interventions and reopening process, focusing on the coevolution of epidemic and awareness during COVID-19 outbreak. Through an empirical analysis based on large-scale data and simulation based on a metapopulation and multilayer network model, we ascertain the impact of human movements and awareness diffusion on the epidemic, elucidate the inherent patterns and effective interventions of different epidemic prevention methods, and highlight the crunch time of each measure. The results are also employed to analyze COVID-19 evolution in other countries so as to find unified rules in complex situations around the world and provide advice on anticontagion and reopening policies. Our findings explain some key mechanisms of epidemic prevention and may help the epidemic analysis and decision-making in various countries suffering from COVID-19.
\end{abstract}

\section{Introduction}

Catching the world by surprise, the novel coronavirus (COVID-19) pandemic has hit over 210 countries and regions, affected more than seven billion people on the planet, and claimed over 480,000 precious lives [1]. Countries around the globe are experiencing different stages of the epidemic; e.g., the ongoing pandemic in Italy is almost under control, while Brazil is going through an outbreak $[2,3]$. As one of the earliest countries challenged by COVID-19, China effectively mitigated the spread of this novel disease, shortened the duration of new cases increasing precipitously, and took appropriate action to promote economic recovery while ensuring the epidemic curve leveling off $[4,5]$. In the battle against COVID-19, China has built much experience, from lockdown restrictions to awareness reminders, from anticontagion policies to reopening measures [6-8]. However, three months after the epidemic was stably controlled in China, new cases in Beijing grew significantly again in mid-June. In this context, some questions arise naturally: Why could China be successful in stopping the pandemic? What led to the recent resurgence of the epidemic in Beijing?

A team organized by the World Health Organization (WHO) gives an unequivocal report of the pandemic in China, suggesting that China's bold approach has changed the course of a rapidly escalating and deadly epidemic and hopes China's successes will encourage other countries to act $[9,10]$. To discuss the experience of China and the relevance between policies in various countries, we investigate the impact of some epidemic factors. We highlight the key regions and crunch time, discuss the patterns and effective measures of the outbreak, and provide advice based on China's experience, which may benefit some parts of the world suffering from COVID-19. 
To analyze COVID-19, some research builds epidemic models to simulate its spread between individuals [11-13] or utilizes statistical and econometric methods to empirically evaluate the impact of policies on the growth rate of infections $[14,15]$. However, previous works neglected the coupling effect between multiple spreading processes in the epidemic. Actually, in complex social systems, diffusion usually does not occur independently but is coupled with each other and evolves together [16-18]. In the case of COVID-19, as the virus spreads among the crowd, information about precautionary measures also circulates simultaneously. These spreading processes affect each other and will both be promoted when the state goes back to work from antiepidemic status. Therefore, it is necessary to understand the interaction of multiple dynamic processes and provide comprehensive solutions for both epidemic prevention and economic recovery.

This work focuses on the coevolution of epidemic spreading and awareness diffusion, taking into account the heterogeneous population mobility, imported cases, and other practical factors. It makes empirical analysis and develops a metapopulation and multilayer network model that leverages lots of real-time mobility and case data. We find evidence of the critical time point for each anticontagion method, e.g., movement restriction and awareness reminder. We also summarize effective interventions that have been proved to achieve large and beneficial health outcomes in China. These findings may benefit the epidemic analysis and decision-making in the rest part of the world. The rest of this paper is organized as follows. In Section 2, we introduce the data resources and interpret our network model and its operating mechanism. In Section 3, we elucidate the results based on simulation and empirical analysis. More discussion can be found in Section 4.

\section{Data and Model}

2.1. Data Description. We carry out the research with large amounts of fine-grained real data, including (i) the registered population and resident population of each prefecturelevel administrative area, extracted from the 2019 statistical yearbook of each province in China (http://www.stats.gov. $\mathrm{cn} / \mathrm{tjsj} /$ ); (ii) the passenger volume carried by modes of transportation, i.e., waterway, highway, railway, and civil aviation, obtained from the statistics released by the Ministry of Transport (http://www.mot.gov.cn/); (iii) the population migration index, migration destination, and local travel intensity of each prefecture-level administrative area, coming from the Baidu Migration (https://qianxi.baidu. com/2020/); (iv) the cumulative number of confirmed cases and the number of imported cases, derived from the Health Commissions (http://www.nhc.gov.cn/xcs/xxgzbd/ gzbd_index.shtml); (v) measures and timing of epidemic prevention and reopening policy on a country-by-country basis. These data are used in simulation and verification, as well as empirical analysis.
2.2. Model. Epidemic models aim to depict the spread of diseases among people, which can be divided into SIS, SIR, SEIR, and other models according to the characteristics of the epidemic [19-23]. The outbreak of COVID-19 in China has the following properties:

(i) Virus carriers have an incubation period of up to two weeks

(ii) At the early stage of the epidemic, the population movement within and between regions amplified localized outbreaks of disease into widespread epidemics, whereas in the latter stage, the new cases increasing mainly results from the imported cases

(iii) In the process of epidemic prevention, there is a continuous and high-intensity reminder of selfprotection awareness and knowledge

The above factors also play an important role in the COVID-19 outbreak in various countries.

Based on these facts, we develop an aggregate model to reproduce the COVID-19 outbreak, which combines a metapopulation network and two-layer coupling networks (Figure 1). This model includes multiple practical factors, i.e., heterogeneous human movements, disease spreading, awareness diffusion, and case importation from abroad. We first establish a metapopulation network to describe the human migration between regions [24-26]. The network includes 345 nodes, i.e., 345 subpopulations, and each node represents a prefecture-level administrative unit in China (Figure 1(a)). The edges depict the individual diffusion between administrative units, and the weights of edges are described by the volume of real population movements between regions at high resolution. Hence, this network draws macroscenarios of the spatiotemporal distribution of COVID-19 in China.

Secondly, a two-layer coupling network is built for each subpopulation to portray the coevolution of epidemic and awareness within each city (Figure 1(b)), where nodes represent individuals $[17,27,28]$. The virtual contact layer describes the awareness diffusion based on the UAU model, and each node in this layer has two possible states: Unaware $(U)$, representing that the individual has no self-protection awareness due to objective or subjective reasons, and Aware $(A)$, referring to individuals with awareness of protecting themselves from this disease [29]. By contrast, the real contact layer supports the epidemic spreading, where each node may be one of the following four states: Susceptible $(S)$, Exposed $(E)$, Infected $(I)$, or Removed $(R)$ according to the classic SEIR model [30]. There is a one-to-one correspondence of nodes between the two layers. The real state of each node is the combined result, so the coupling network consists of up to seven kinds of nodes $Z_{i}=\left[U S_{i}, U E_{i}, U R_{i}, A S_{i}, A E_{i}, A I_{i}, A R_{i}\right]$, e.g., aware infected node $(A I)$ and unaware susceptible node (US). Here, we consider that once an individual becomes infected, he or she will not ignore this disease anymore, which indicates the absence of UI state [31]. Edges in the virtual contact layer represent conscious interactions caused by online or offline communication between individuals, while edges in the real contact layer represent infections caused by actual contact 
between individuals. The coupling effect is reflected by the awareness-dependent exposure probability, which means that aware people $\left(\beta^{A}\right)$ are less likely than unaware people $\left(\beta^{U}\right)$ to be exposed to dangerous infectious environments, i.e., $\beta^{A}=\gamma * \beta^{U}(\gamma<1)$.

Based on the model described above, the simulation experiments run as follows [25], with each round of evolution including two steps:

(1) In the first stage, we count the individual diffusion between subpopulations and the overseas imports. Then, we update the total population and proportion of individuals in each state for every subpopulation; e.g., the total number of residents in the $i^{\text {th }}$ subpopulation at $(t+1)^{t h}$ time step is $Z_{i}(t+1)=Z_{i}(t)+\Delta Z_{i}(t)$.

(2) In the second stage, epidemic and awareness evolve together within subpopulations. We first calculate the effective exposure rate as follows:

$$
\beta_{i}^{e f f}(t)=\text { inRate } * \beta * \frac{N_{i}^{A I}(t)}{Z_{i}(t)}
$$

where inRate is the local travel intensity within regions, $\beta$ is the basic exposure rate, and $N_{i}^{A I}(t)$ and $Z_{i}(t)$ are the number of infected (AI) and total population in the $i^{\text {th }}$ subpopulation at $t^{\text {th }}$ time step, respectively. Therefore, inRate $*\left(N_{i}^{A I}(t) / Z_{i}(t)\right)$ indicates an individual's possible exposure to infected individuals. Given that $\beta$ is the exposed probability of an individual in a single contact with the infected population, $\beta_{i}^{\text {eff }}(t)$ depicts the actual exposed probability of an individual in possible several contacts with the infected population of the $i^{t h}$ subpopulation at $t^{\text {th }}$ time step. Next, the probability of an individual being in a certain state at $(t+1)^{\text {th }}$ time step is updated as follows:

$$
\begin{aligned}
& P_{i}^{U S}(t+1)=\left[P_{i}^{U S}(t) *(1-\lambda)+P_{i}^{A S}(t) * \delta\right] *\left(1-\beta^{U}\right)+\left[P_{i}^{U R}(t) *(1-\lambda)+P_{i}^{A R} * \delta\right] * \eta, \\
& P_{i}^{U E}(t+1)=\left[P_{i}^{U S}(t) *(1-\lambda)+P_{i}^{A S}(t) * \delta\right] * \beta^{U}+\left[P_{i}^{U E}(t) *(1-\lambda)+P_{i}^{A E} * \delta\right] *(1-\alpha), \\
& P_{i}^{U R}(t+1)=\left[P_{i}^{U R}(t) *(1-\lambda)+P_{i}^{A R}(t) * \delta\right] *(1-\eta)+P_{i}^{A I}(t) * \mu * \delta, \\
& P_{i}^{A S}(t+1)=\left[P_{i}^{U S}(t) * \lambda+P_{i}^{A S}(t) *(1-\delta)\right] *\left(1-\beta^{A}\right)+\left[P_{i}^{U R}(t) * \lambda+P_{i}^{A R} *(1-\delta)\right] * \eta, \\
& P_{i}^{A E}(t+1)=\left[P_{i}^{U S}(t) * \lambda+P_{i}^{A S}(t) *(1-\delta)\right] * \beta^{A}+\left[P_{i}^{U R}(t) * \lambda+P_{i}^{A R} *(1-\delta)\right] *(1-\alpha), \\
& P_{i}^{A I}(t+1)=\left[P_{i}^{U E}(t)+P_{i}^{A E}(t)\right) * \alpha+P_{i}^{A I}(t) *(1-\mu), \\
& P_{i}^{A R}(t+1)=\left[P_{i}^{U R}(t) * \lambda+P_{i}^{A R}(t) *(1-\delta)\right] *(1-\eta)+P_{i}^{A I}(t) *(1-\delta) * \mu,
\end{aligned}
$$

where $\lambda$ is the awareness diffusion rate, $\delta$ is the awareness forgetting rate, $\alpha$ is the infection rate, $\mu$ is the removal rate, and $\eta$ is the reinfection rate. These probabilities can be utilized to calculate the population of each state in the $i^{\text {th }}$ subpopulation at the $(t+1)^{\text {th }}$ time step.

\section{Results}

3.1. Epidemic Simulation. By employing our model to simulate the outbreak, we reproduced the spread of COVID19 in China from January 24th (i.e., the Chinese New Year's Eve) to mid-April. The parameter set, which makes the simulation result closest to the real final cumulative number of cases, is selected, such that the epidemic is therefore explained well by the simulation. We extract the difference between the simulation results and real data of each province (except Hong Kong, Macao, and Taiwan) for further analysis (Figure 2(a)).

These differences suggest a segregation effect since the results in eastern and southwestern China differ significantly. Hence, we map the data to the real geographic location (Figure 2(b)). According to the map, we take the antiepidemic achievements of some municipalities as the benchmark, i.e., Beijing, Tianjin, and Shanghai. It is found that the severity of the epidemic in the central region and east coast, as well as the Heilongjiang province, is relatively high (the red region in Figure 2(b), representing the places where the simulation results are lower than the real data). Nevertheless, the severity of the epidemic in the southwest region is relatively low (the blue region in Figure 2(b), representing the places where the simulation results are higher than the real data).

To some extent, this phenomenon indicates that the spread of COVID-19 is lower than expected in southwest China while higher in central and eastern China. This might be due to multiple reasons. On the one hand, given the low population density in the western and southern regions, the epidemic spreading is relatively difficult; on the other hand, regions with frequent overseas trade and population transfer, e.g., the east coast and Heilongjiang, are particularly affected by the international outbreak.

3.2. Key Factors Analysis. In the model setup, we consider the impact of human movement (including population mobility within cities and the population migration between cities) and awareness diffusion on the epidemic. The Baidu Migration website provides population mobility indexes to show the volume of population movement, and the 


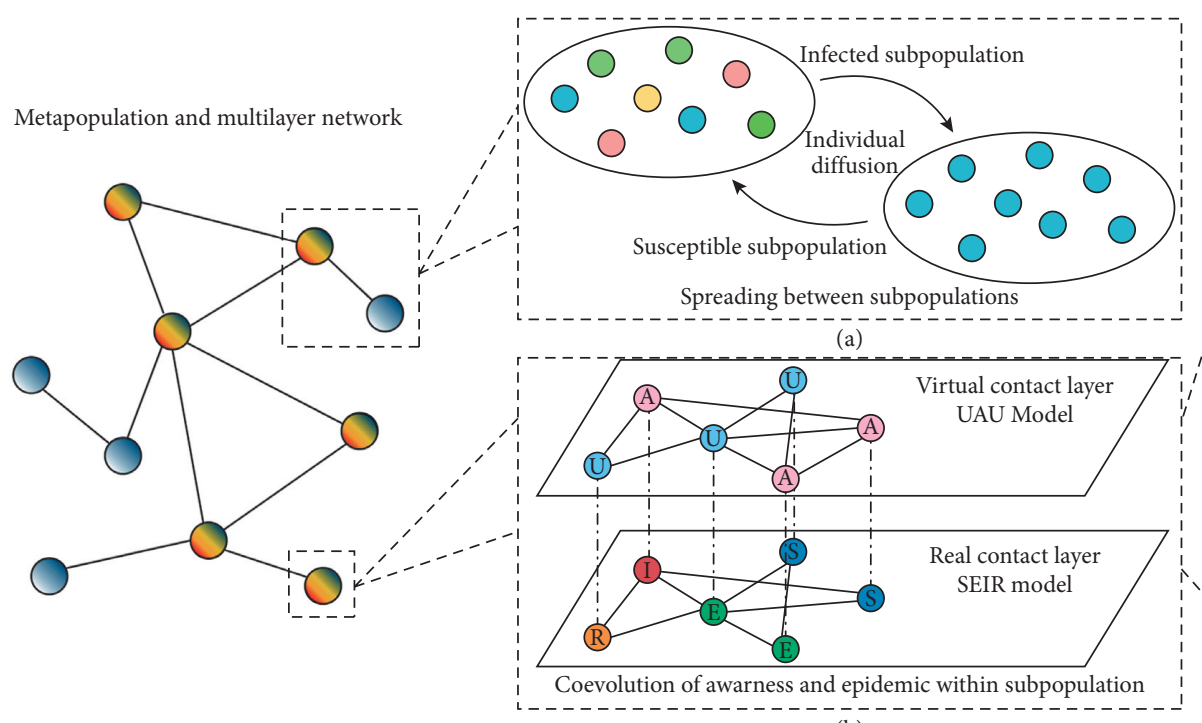

(b)

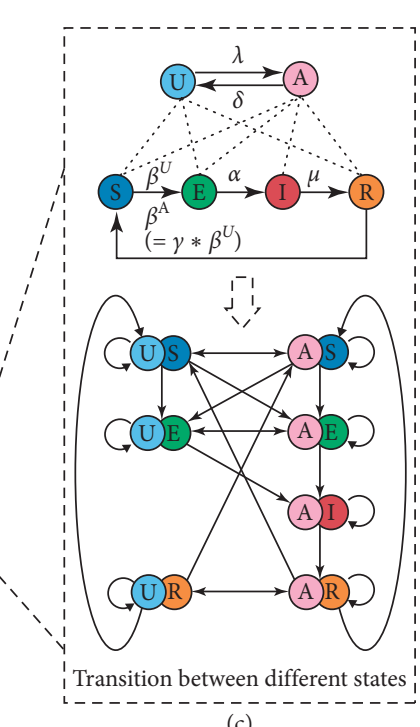

(c)

Figure 1: A simple illustration of our model framework. The left part displays the metapopulation network, where the blue and rainbow-colored nodes represent the susceptible and infected subpopulations, respectively. Subfigure (a) represents the individuals of each subpopulationFigr moving between different subpopulations along with the epidemic and awareness. Subfigure (b) demonstrates the awareness diffusion (UAU model) and epidemic spreading (SEIR model) interaction based on virtual or real individual contacts within each subpopulation, modeled by a multilayer coevolution network. (c) indicates the mechanism of transition between different states based on several parameters. (a) Spreading between subpopulation. (b) Coevolution of awareness and epidemic within a subpopulation. (c) The transition between different states.

comparison of these indexes indicates the change of population flow in different regions or periods. We adjust these key factors and try to understand how these factors affect epidemic prevention. To be specific, we make the following adjustments to the three factors: (1) global adjustment for all regions and periods; (2) local adjustment, especially adjustments in Hubei province; (3) temporal adjustment in the early, middle, and later periods.

\subsubsection{Data Analysis and Impact of Factors}

(1) Factor One: Population Mobility within Cities. First, comparing real-time mobility data on a year-by-year basis can illustrate how the population mobility within each city is controlled. The data suggest that the average population mobility within cities was limited to $81.3 \%$ of the same period last year. There is a power-law exponent of around 0.96 between the 2020 and 2019 population mobility indexes. Regions with significantly strong restrictions include Hubei and Xinjiang provinces, which were controlled at around $58.8 \%$ (Figure 3(a)). Next, we adjust the actual population mobility data in China from the above three aspects: global control, local control in Hubei province, and temporal control (Figure 3(b)). Results indicate that global control causes the most dramatic changes in the epidemic, followed by the control in Hubei. Both of these controls lead to an exponential change in the cumulative cases. It can be found that the population movement control within Hubei, the worst-hit area in China, can produce an important effect on the evolution of COVID-19.

(2) Factor Two: Population Migration between Cities. Similarly, we identify that the average population migration between cities was limited to $60.6 \%$ of the same period last year. The power-law exponent between the 2020 and 2019 population migration indexes is about 0.91 . Beijing, Hong Kong, and Hubei provinces imposed strict restrictions, limiting the migration to between $22.2 \%$ and $45.4 \%$ (Figure 3(c)). This evidence elucidates that regional governments have taken much tougher measures to control the migration between cities than mobility within cities. Results also show that the control at the early stage has a comparable influence on the epidemic with the global control (Figure 3(d)). This phenomenon underlines the necessity and effectiveness of early intervention in the management of population migration between cities.

(3) Factor Three: Awareness Diffusion. We study three model parameters related to self-protection awareness, i.e., the rate of awareness diffusion, the rate of awareness forgetting, and the proportion of awareness in the initial configuration. The experimental results suggest that adjusting the awareness diffusion rate makes the greatest impact on the epidemic, compared with the other two (Figure 3(e)). It can be judged by the parameter value that self-protection awareness has been widely spread during the COVID-19 outbreak in China. The awareness diffusion rate is selected as the main variable for controlling the awareness spreading. We find that the earlier we take action, the more remarkable the change in the epidemic will be (Figure 3(f)). This phenomenon also applies to the previous two factors. The result of awareness ascertains the important role played by self-protection awareness, which is created by publicity and information dissemination, in the war against the pandemic.

(4) Comparison of Factors. We further make a comparative analysis of the above three factors. On the basis of China's 


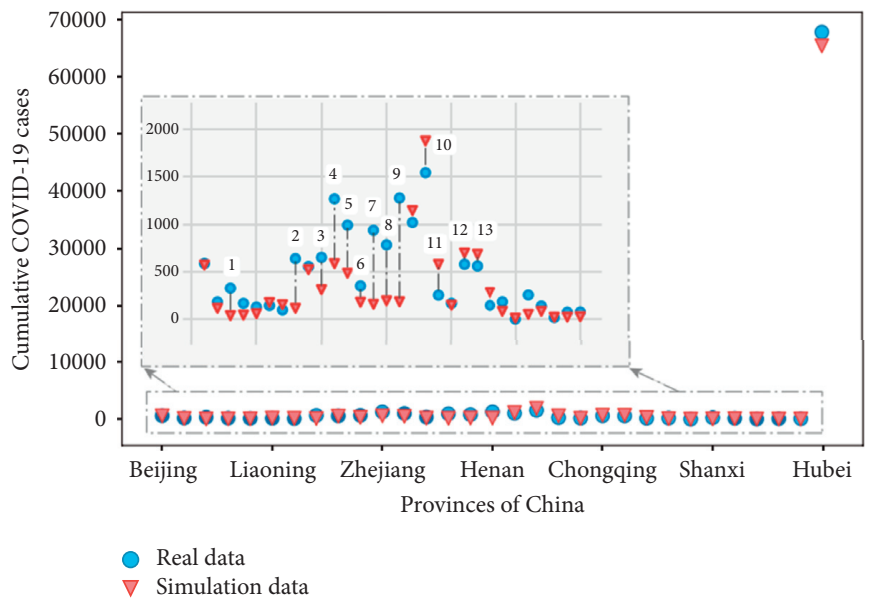

(a)

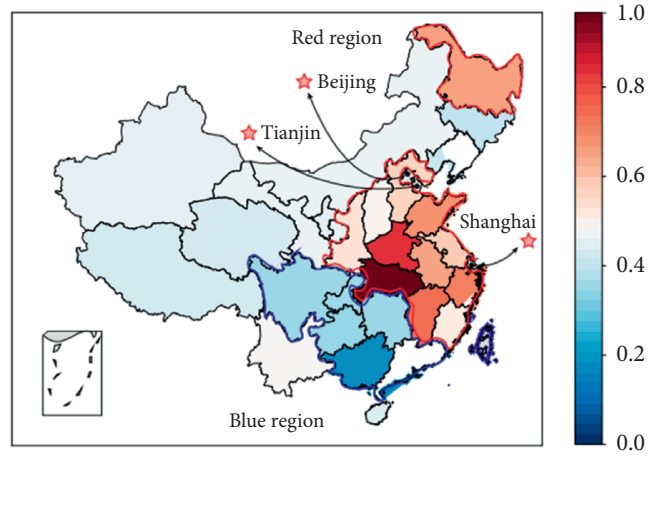

(b)

Figure 2: Comparison of simulation results and real epidemic data in China. In subfigure (a), the blue circles and red triangles represent the real and simulated cumulative numbers of confirmed cases in Chinese provinces on April 12th, respectively, with 31 provinces in total ranked by administrative code (except Hong Kong, Macao, and Taiwan). A partial enlarged view of the dotted rectangle highlights the data from 30 provinces except Hubei. In subfigure (b), the differences between real and simulated cumulative confirmed cases were converted into a heat map to observe the geographical distribution of the epidemic. The red region indicates that the real data is higher than the simulation result, while the blue region indicates the opposite. (a) Simulation results vs. real data. (b) Distribution in India.

practical measures, it brings about the most dramatic change in the epidemic to adjust the population mobility with cities, followed by awareness spreading and population migration between cities (Figures 3(b)-3(f)).

On the one hand, the results confirm the lockdown restrictions between regions in China. Actually, China attached great importance to avoiding the hidden risk of the epidemic transferring among cities and strictly confined the human movement between cities. Therefore, the reopening policy began within cities, and college students were prohibited from returning to school to avoid mass migration across regions. On the other hand, the results emphasize the importance of controlling population mobility within cities and anticontagion awareness. The human activities within each region in China were limited by mandatory stay-athome order, closure of physical business premises, and cancellation of nonessential gatherings. China also conducted anticontagion publicity through various channels, e.g., mainstream media, We media, and slogans, to improve the awareness of self-protection and knowledge of residents.

We also study the effect of factor adjustment in key areas, i.e., Hubei province in this case, which is the worsthit area in China. Results show that the population mobility within Hubei leads to greater changes in the epidemic than population migration from this province (Figure 4(a)). This indicates that there was room for improving the human movement control within Hubei, whereas the population outflow from Hubei has been fully restricted, which is confirmed by actual data (Figure 4(b)). Therefore, it is reasonable to believe that if the population movement in Hubei is more strictly confined during the outbreak, the epidemic in China will be more effectively mitigated.

On the whole, we adjust the three key factors by global, local, and temporal adjustments. Results show the crucial influence of these factors on the epidemic. Although many people emphasize the travel restriction between regions, equal attention should be given to population mobility control within each region and awareness reminders for the residents. We highlight the importance of measures in Hubei, especially controlling the population mobility within this region, and evaluate the epidemic prevention in Hubei through empirical analysis. We also find that early interventions always do more with less for each factor. Hence, we next conduct more analysis to discover how measures in different stages affect the epidemic and what effective measures and crunch time exist.

3.2.2. Anticontagion and Reopening Policies. In the light of the above findings, we further examine the three factors in detail to discuss specific crunch time and effective measures. We focus on the impacts of them on the epidemic at different periods and for different goals, i.e., epidemic prevention and economic recovery. Since we regard each week as a period, the simulation duration from late January to mid-April is divided into 11 periods. The results reveal that the control of each factor has a critical time point. If we do not take the crunch time seriously, it is highly likely that a large-scale outbreak of pandemic will occur.

(1) Factor One: Population Mobility within Cities. We first simulate the reopening timetable by implementing the resumption policy from a certain period in turn. It is shown that if work resumes within cities earlier than the fifth week when the new cases almost decay to zero, the previous anticontagion achievements will be offset, leading to an outbreak (Figure 5(a)). Actually, China went back to work from around February 23th, which is just in the fifth week. This timing was exactly consistent with the simulation result. 


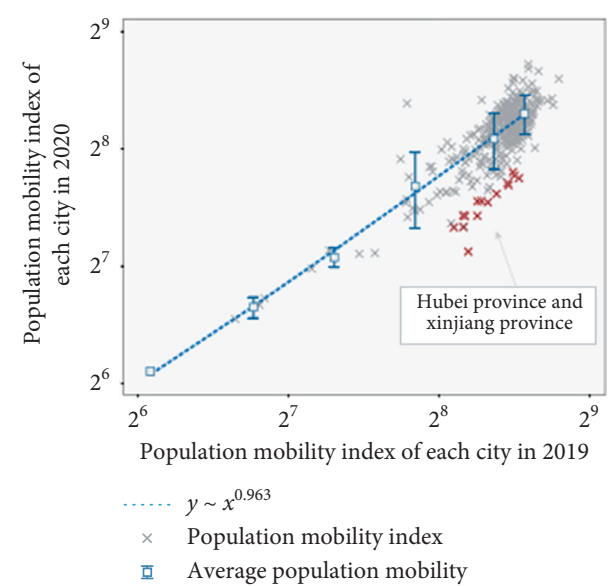

(a)

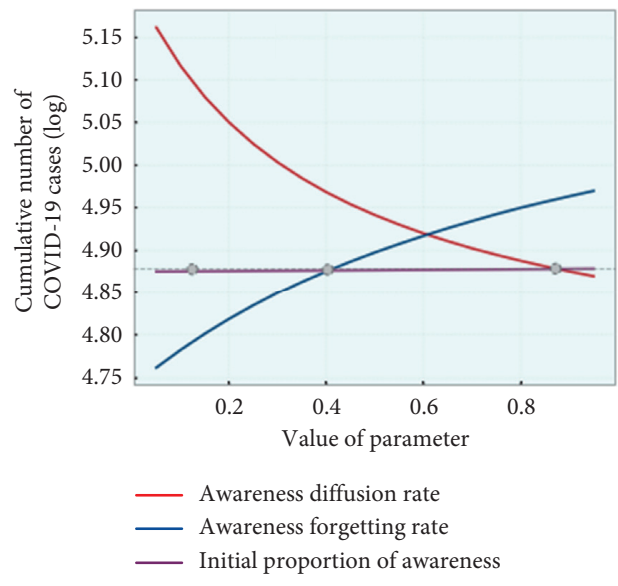

(c)

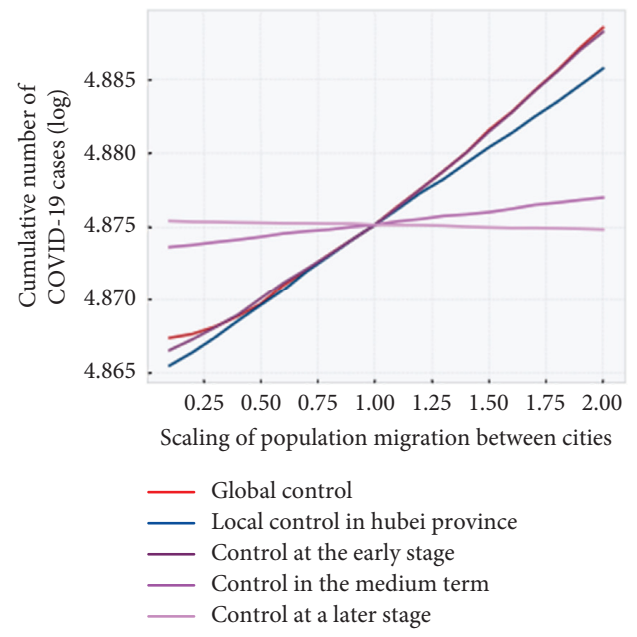

(e)

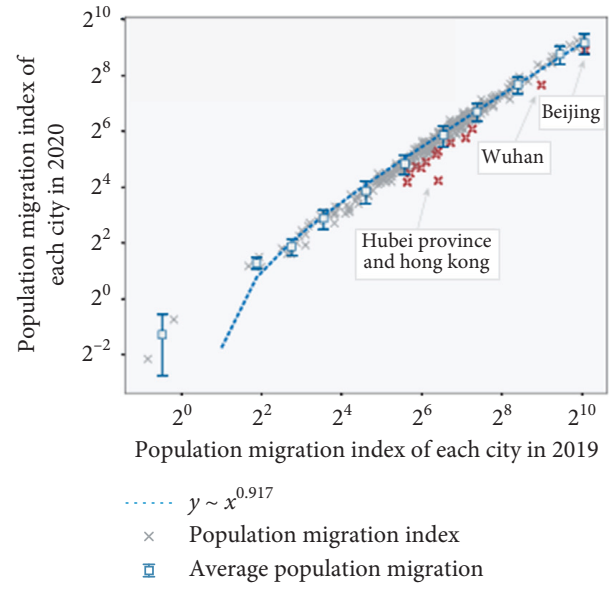

(b)

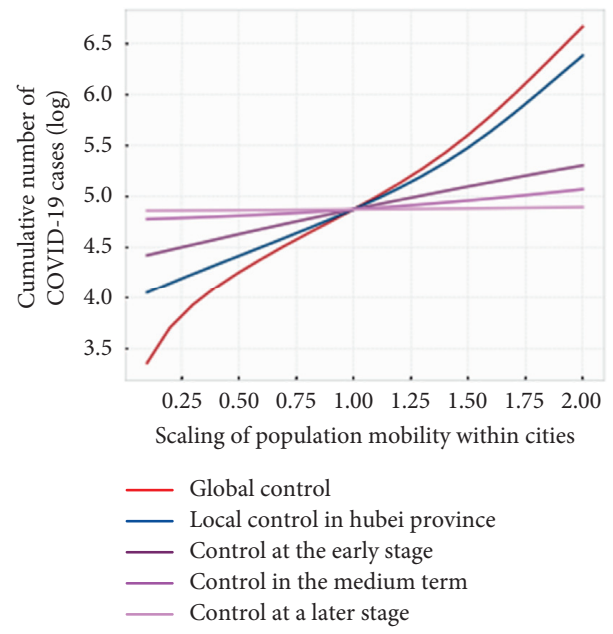

(d)

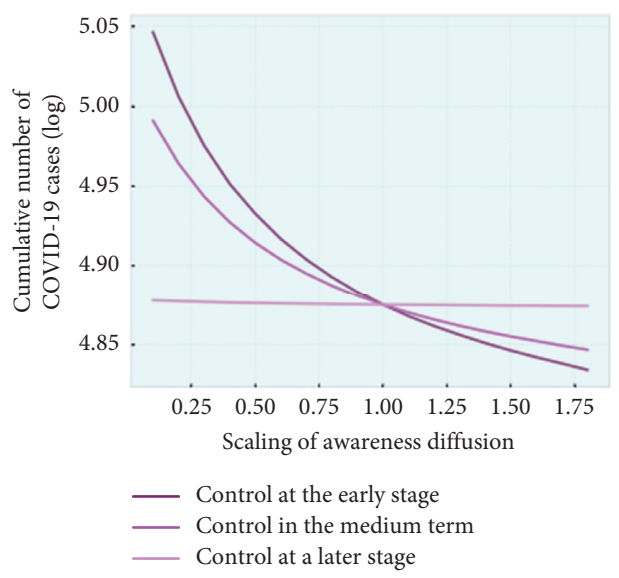

(f)

FIgURE 3: Real data analysis and the impact of three key epidemic factors, i.e., population mobility within cities, population migration between cities, and awareness diffusion. Subfigure (a) demonstrates the comparison between the 2019 and 2020 population mobility index of each city with a fitted parameter of 0.96 . Subfigure (b) displays that the simulated cumulative confirmed cases change along with the control measures of population mobility within cities. The abscissa indicates the scaling factors. That is, the actual volume is used as the standard to zoom in or out. We perform the global, local, and temporal controls on an actual basis, with scaling ratios ranging from 0.1 to 2.0. Subfigures (c) and (d) show the results of controlling the population migration between cities in the same way. Subfigure (e) reveals the impact of three model parameters affecting awareness diffusion. The grey dots record the value of each parameter used in the model. Subfigure (f) demonstrates the outcomes of applying temporal control to awareness diffusion. (a) Data of population mobiliy within cities. (b) Data of population migration between cities. (c) Parameter of awareness diffusion. (d) Impact of population mobility within cities. (e) Impact of population migration between cities. (f) Impact of awareness diffusion. 


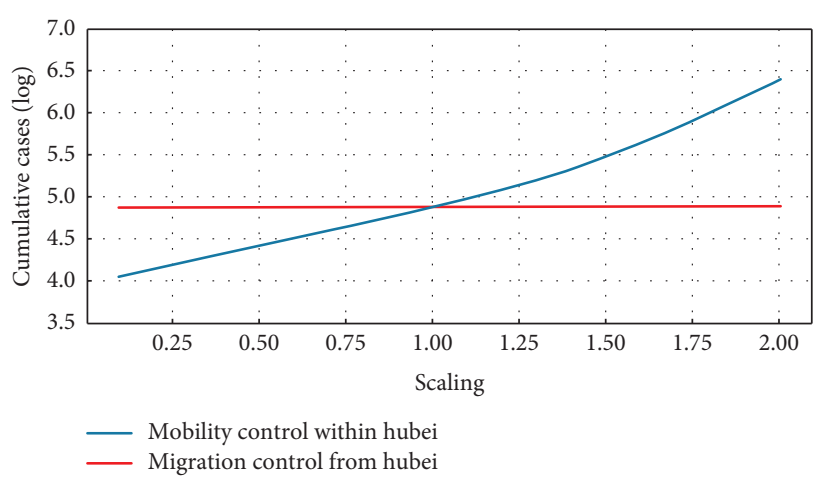

(a)

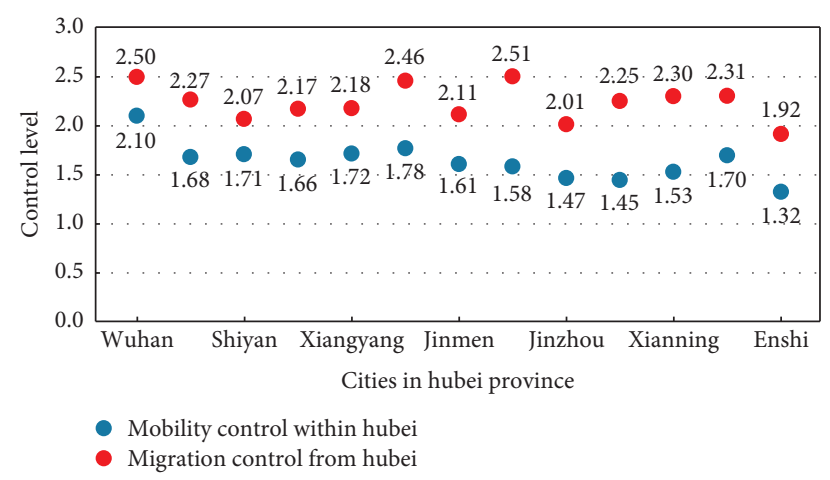

(b)

FIgURE 4: Comparison between the impacts of different controls in Hubei province. Subfigure (a) provides the simulation results of controlling the population mobility within Hubei (blue line) and migration from Hubei (red line) on a practical basis, with scaling ratios ranging from 0.1 to 2.0. In subfigure (b), the blue and red dots represent the real control level of population mobility within Hubei and migration from Hubei, respectively, which is calculated as dividing the 2019 average index by the 2020 average index of the same period. The real data identifies the much more strict restriction on population migration from Hubei than mobility within Hubei, which is consistent with the simulation results. (a) The simulation results of Hubei province. (b) Real data of Hubei province.

Therefore, the reopening within cities of China is reasonable and has effectively promoted economic recovery and epidemic prevention. Next, the epidemic prevention timetable shows the results of restricting human mobility within cities from a certain period in turn. We find the critical time appears in the fourth week, and if the population mobility restriction misses this time point, the intervention will have little effect (Figure 5(a)).

In addition, we investigate the impact of stricter prevention, which means taking tougher control measures on human movement within cities than the actual policy from a certain period in turn. As a result, only the strict restriction started within the first three weeks is efficient at slowing the growth substantially (Figure 5(a)). If we aim to reduce the final cumulative confirmed cases in China to less than half of the real data (i.e., less than 40,000 ) by controlling the population mobility within cities, the action should be taken in the first two weeks, i.e., limiting it to $76 \%$ of the current control level from the first week, or $64 \%$ from the second week (Figure 5(b)).

(2) Factor Two: Population Migration between Cities. The analysis of migration between cities is conducted in the same way as above. We find that the critical time points of deploying and lifting the migration restrictions between cities are the third and second week, respectively (Figure 5(c)). It means that we should take action to restrict the migration across regions at least before the explosive growth of new cases and restart it after the new cases drop to half the peak. Since these time points are earlier than the previous factor, it again supports the conclusion that we should pay more attention to the early control of migration between cities. Results also reveal that the stricter restriction on migration across regions should start from the first week to effectively mitigate the outbreak (Figure 5(c)). However, the unprecedented migration confined in China is almost sufficiently strict. Therefore, if only tightening it further, the final cumulative confirmed cases will not be cut by half (Figure 5(d)).
(3) Factor Three: Awareness Diffusion. Similarly, the experiments on awareness show that we should start an awareness reminder no later than the third week and wait until at least the fourth week before proceeding to relax the publicity of anticontagion awareness (Figure 5(e)). It reveals that we should always value the awareness reminder at least until the new cases decline to near zero. If we try to limit the epidemic more efficiently, more efforts need to be put into publicizing awareness for the first three weeks. By contrast, the decline of the epidemic caused by the awareness reminder is weaker than its increase, which means that the self-protection awareness of Chinese residents is relatively strong. On this basis, it is not achievable to bring the cumulative cases below 40,000 by only controlling awareness spreading (Figure 5(f)).

(4) Summary of Comparison. In general, population mobility within cities, migration between cities, and awareness diffusion are proven to be key epidemic factors. Taking appropriate measures from these aspects at the right time can effectively control the pandemic (Figures 5(a)-5(e)). The controlling of these factors changes the course of a rapidly escalating and deadly epidemic, and the crunch time for epidemic prevention is discussed above. We also obtain consistent evidence that countries should reopen at least after the new case drops to half the peak.

However, once a state reopens, these human activity and awareness factors may lead to a resurgence of the epidemic at any time. The sudden resurgence of COVID-19 in Beijing in mid-June has refocused attention on epidemic prevention and control. In the previous three months, the country gradually resumed work and classes, and large-scale gatherings and movements occurred again. The anticontagion awareness reminder to the public also slackened. As a result, these conditions provide an opportunity for the epidemic to spread. Fortunately, Beijing immediately stopped resuming work, restricted population movement, updated the public on the epidemic information, and conducted a large number 


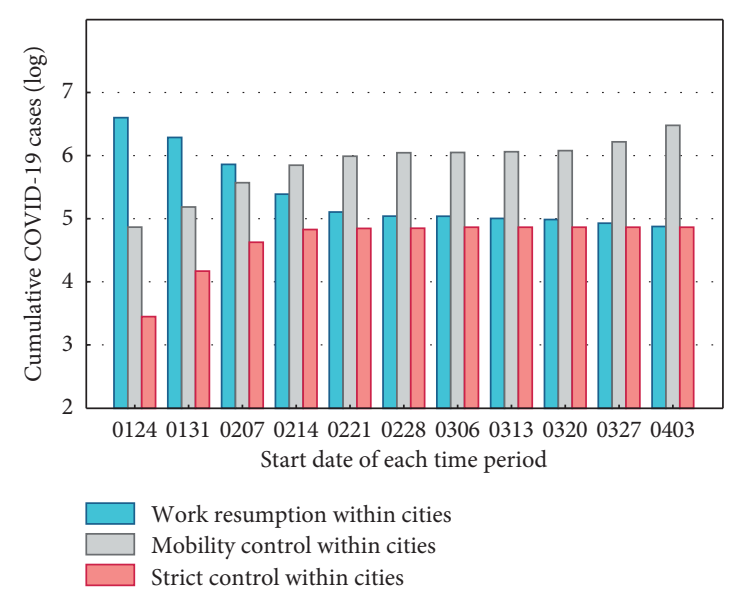

(a)

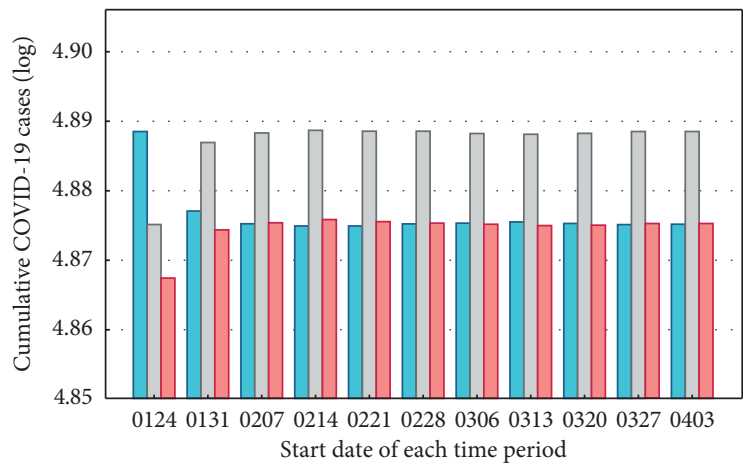

Work resumption between cities Migration control between cities Strict control between cities

(c)

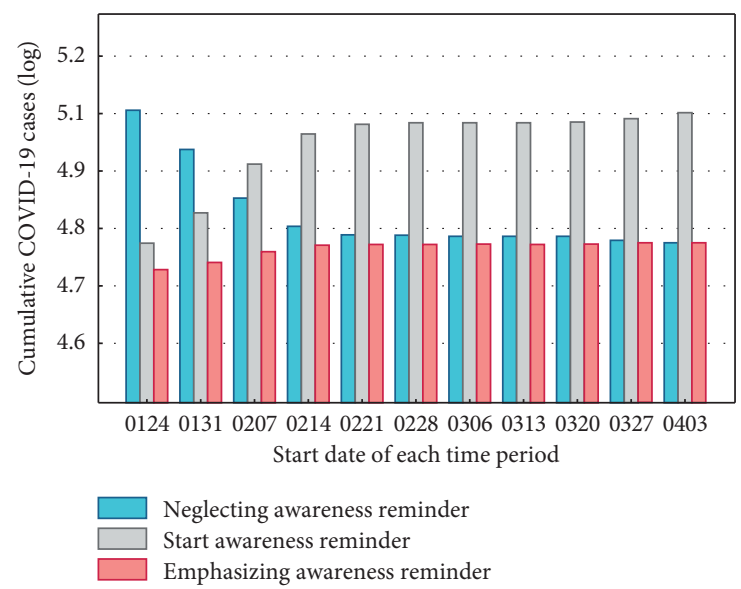

(e)

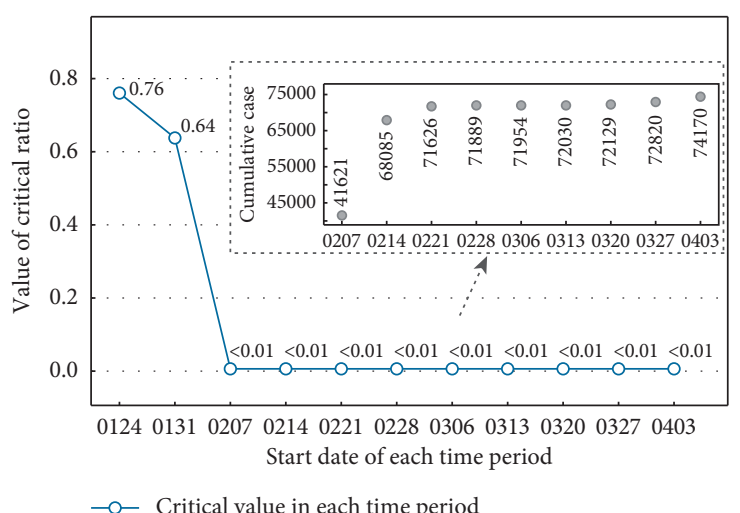

(b)

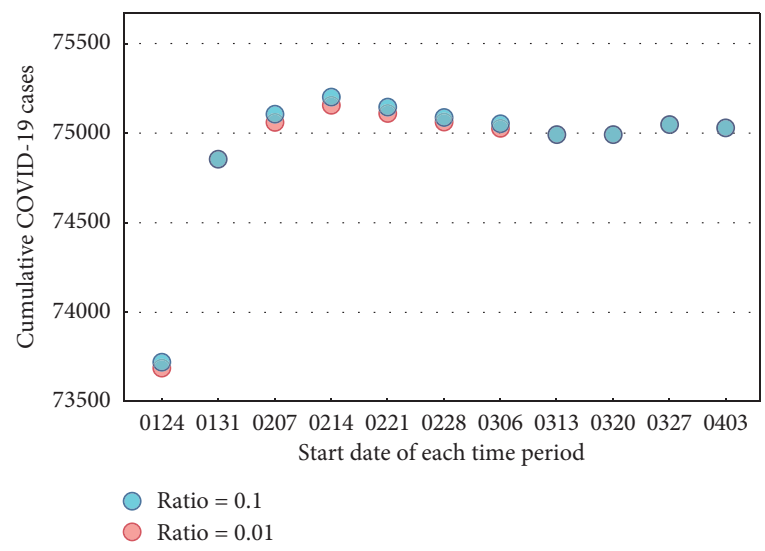

(d)

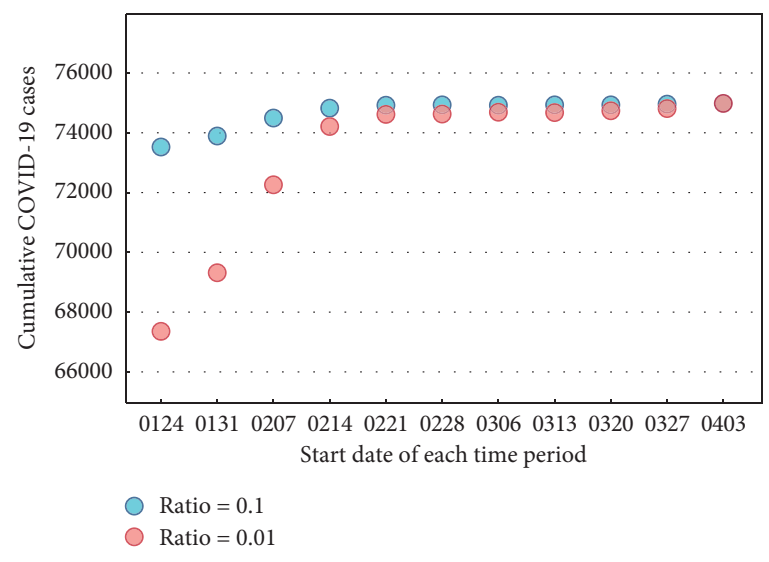

(f)

FIgURE 5: Timetable for the work resumption, epidemic prevention, and strict restrictions. (a) records the simulated cumulative confirmed cases, when the state goes back to work within each city (blue bar), restricts the population mobility within cities (grey bar), and takes tougher control measures on mobility within cities (red bar) since different periods. (c) shows the results of controlling the population migration between cities in the same way. The blue, grey, and red bars in (e) demonstrate neglecting, starting, and emphasizing the awareness reminder, respectively. To cut the final cumulative confirmed cases by half, (b) shows the critical limiting ratio of population mobility within cities. The partial enlarged view in (b) documents the cumulative cases when this factor is adjusted to $1 \%$ of the true level, which shows that the goal cannot be achieved in the third week or beyond. Since the goal to cut cumulative cases by half also cannot be achieved by only controlling the other two factors, i.e., the population migration between cities and awareness diffusion, (d) and (f) document the impact of limiting them to $1 \%$ and $10 \%$ of the true level. (a) Timeline for population mobility management. (b) Timeline for population migration management. (c) Timetable for awareness diffusion. (d) Critical value of population mobility management. (e) Critical value of population migration management. (f) Critical value of awareness diffusion. 
of viral tests, which quickly curbs the growth of coronavirus cases.

3.3. Experience of China and Reality of Various Countries. On the basis of the above analysis, we summarize the characteristics and experience of China in epidemic prevention and economic recovery:

(1) The epidemic prevention started at the initial stage of the outbreak when new cases just began to increase exponentially. The key measures included restrictions on population mobility within cities, restrictions on population migration between cities, and publicity of anticontagion awareness.

(2) The reopening policy was implemented when the pandemic almost was under control, i.e., new cases decreased almost to zero. The resumption of work started within cities and then extended to human movements across cities.

(3) Large-scale new cases in China lasted about one month.

The war against COVID-19 of China has gained remarkable achievements. Based on China's experience in the epidemic prevention and reopening process, we analyze the epidemic policies in various countries, where we focus on the deploying and lifting of restrictions on population movement. The results are presented in Figure 6, and the main findings are listed as follows:

(1) Some Asian countries, e.g., Japan and South Korea $[32,33]$, also started anticontagion measures at the beginning of the outbreak and resumed work when the epidemic is under stable control (Figure 6(a)). The outbreak was short-lived in these countries.

(2) In some European countries, e.g., the United Kingdom, Italy, and Germany [34, 35], the epidemic prevention started relatively late, with a time lag of about two weeks from the initial outbreak, and the reopening measures took effect when the epidemic was initially under control (Figure 6(b)). For example, in the early stages of the pandemic, the UK adopted loose anticontagion measures, delayed lockdown restrictions, and the self-protection awareness of the residents was weak. These led to an insufficient implementation of the government's recommendations, such as keeping a social distance, which made the outbreak last longer.

(3) In the American countries, e.g., the United States and Canada [36, 37], anticontagion policies were put forward in the early stage of the outbreak, but the practical measures were not strict enough. The countries got back to work when the outbreak was still in progress. Hence the epidemic lasted for a long time (Figure 6(c)). For instance, in the early days of epidemic prevention in the United States, effective measures such as wearing masks were not emphasized enough by the government and many residents ignored personal protection, resulting in the explosive growth of coronavirus cases. In addition, many states began to lift social distancing measures and organize resumption of production even before the new cases showed a clear downward trend, and due to social causes, large-scale protest marches broke out in many parts of the United States. As a result, rather than being restricted, the population movement has become more active. These complex conditions make it difficult for the US epidemic to be effectively controlled.

Some other countries around the world now are still in epidemic growth, e.g., India, Russia, Mexico, and Brazil [38]. These countries began to prevent the epidemic early, but due to economic pressure and the world environment, they began to resume work during the explosive growth. Therefore, there is a risk of sustained large-scale outbreaks in these countries.

\section{Discussion}

This work investigates the coevolution of the epidemic spreading and awareness diffusion during the COVID-19 outbreak. It develops a network model with large-scale real data of heterogeneous human movements and imported cases to simulate the epidemic. We focus on three key factors of the epidemic, i.e., population mobility within regions, population migration between regions, and awareness diffusion, examining their effective measures and crunch time separately. Results elucidate the important role played by these factors in epidemic prevention and ascertain the impact of measures taken in Hubei on the national outbreak. We also highlight the critical time points for each anticontagion measure.

The experience of China is employed to analyze COVID19 in other parts of the globe, to find unified rules in 275 complex situations around the world and give advice on epidemic prevention. The advice includes avoiding gathering and lack of self-protection awareness; strongly preventing the coronavirus from spreading within key regions or beyond; attaching importance to the crunch time, to eliminate risks at the source, and keeping the pandemic from recurring caused by reopening measures. Our findings explain some key mechanisms of epidemic prevention and contribute to the decision-making in various countries suffering from COVID-19.

We attempt to discuss the impact of awareness diffusion on the COVID-19 pandemic in this paper. Since awareness plays an important role in controlling epidemic spreading, many related issues are worth studying. We hope that this paper will inspire more work on the relationship between the epidemic and awareness and encourage more countries to 


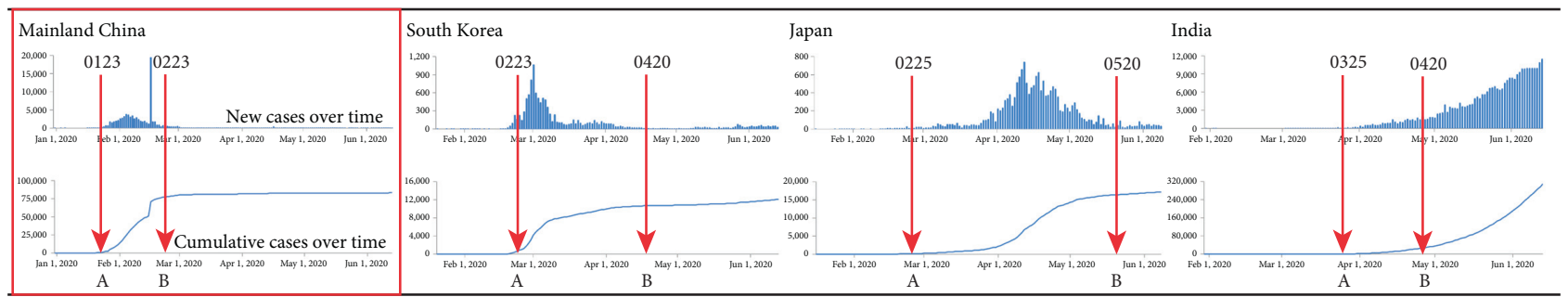

(a)

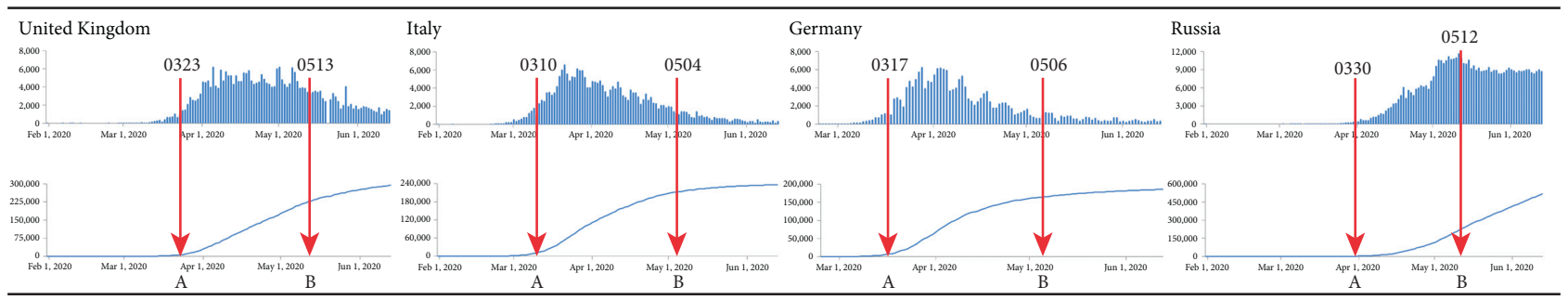

(b)

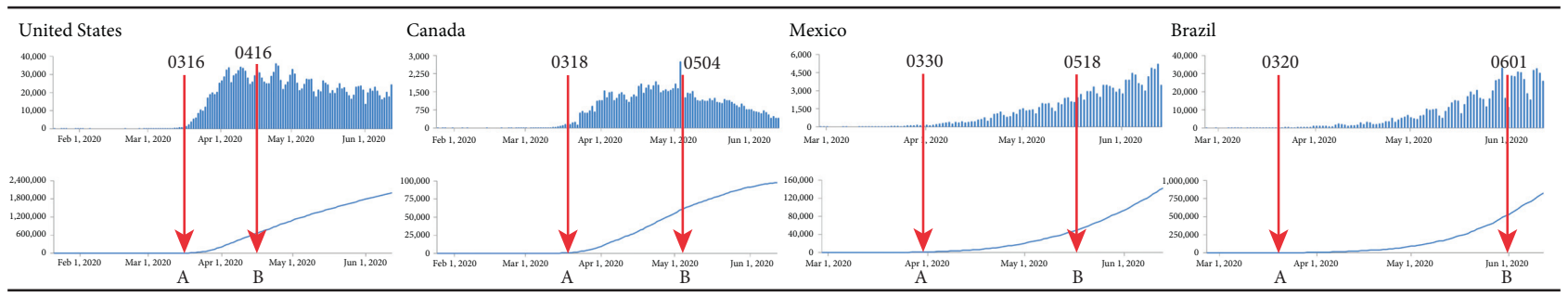

(c)

Figure 6: Anticontagion and reopening policies in various countries. We select 12 countries from Asia, Europe, and America as examples and mark the time points at which they took antiepidemic and reopening measures, corresponding to points A and B in the figure, respectively. We demonstrate the new cases and cumulative cases over time in the COVID-19 outbreak of each country. (a) Asia. (b) Europe. (c) America.

build residents' awareness of epidemic prevention in the battle against COVID-19.

\section{Data Availability}

The data source is described within the manuscript. The data supporting our results can be found in the official government websites of relevant countries and the following websites: http://www.stats.gov.cn/tjsj/, http://www.mot.gov.cn/, https:// qianxi.baidu.com/2020/, and http://www.nhc.gov.cn/xcs/ xxgzbd/gzbd_index.shtml.

\section{Conflicts of Interest}

The authors declare no conflicts of interest.

\section{Authors' Contributions}

Conceptualization was performed by $\mathrm{YF}$ and AZ; formal analysis was carried out by LL, YF, and AZ; LL was responsible for providing software and visualization and original draft preparation. LL and AZ were responsible for editing and supervision.

\section{Acknowledgments}

This work was supported by the National Natural Science Foundation of China (Grant no. 71731002).

\section{References}

[1] I. Ali and M. L. Omar, "Covid-19: disease, management, treatment, and social impact," Science of the Total Environment, vol. 728, Article ID 138861, 2020.

[2] M. Gatto, E. Bertuzzo, L. Mari et al., "Spread and dynamics of the COVID-19 epidemic in Italy: effects of emergency containment measures," in Proceedings of the National Academy of Sciences, vol. 117, no. 19, pp. 10484-10491, 2020.

[3] W. Clare, J. Smith, and R. Morgan, "Covid-19: the gendered impacts of the outbreak," The Lancet, vol. 395, no. 10227, pp. 846-848, 2020.

[4] B. F. Maier and D. Brockmann, "Effective containment explains subexponential growth in recent confirmed covid-19 cases in China," Science, vol. 368, no. 6492, pp. 742-746, 2020.

[5] Z. Wang, J. Wang, and J. He, "Active and effective measures for the care of patients with cancer during the covid-19 spread in China," JAMA Oncology, vol. 6, no. 5, pp. 631-632, 2020.

[6] C. R. Wells, P. Sah, S. M. Moghadas et al., "Impact of international travel and border control measures on the global 
spread of the novel 2019 coronavirus outbreak," in Proceedings of the National Academy of Sciences, vol. 117, no. 13, pp. 7504-7509, 2020.

[7] M. U. G. Kraemer, C.-H. Yang, B. Gutierrez et al., "The effect of human mobility and control measures on the covid-19 epidemic in China," Science, vol. 368, no. 6490, pp. 493-497, 2020.

[8] J. S. Jia, X. Lu, Y. Yuan, Ge Xu, J. Jia, and N. A. Christakis, "Population flow drives spatio-temporal distribution of covid19 in China," Nature, vol. 582, pp. 1-5, 2020.

[9] K. Kupferschmidt and J. Cohen, "Can China's covid-19 strategy work elsewhere?," Science, vol. 367, no. 6482, pp. 1061-1062, 2020.

[10] J. Gao, Y. Yin, B. F. Jones, and D. Wang, "Quantifying policy responses to a global emergency: insights from the covid-19 pandemic," 2020, https://arxiv.org/abs/2006.13853.

[11] M. Chinazzi, J. T. Davis, M. Ajelli et al., "The effect of travel restrictions on the spread of the 2019 novel coronavirus (covid-19) outbreak," Science, vol. 368, no. 6489, pp. 395-400, 2020.

[12] H. Tian, Y. Liu, Y. Li et al., "An investigation of transmission control measures during the first 50 days of the covid-19 epidemic in China," Science, vol. 368, no. 6491, pp. 638-642, 2020.

[13] Y. Liu, T. W. Russell, J. K. Adam et al., "The effect of control strategies to reduce social mixing on outcomes of the covid-19 epidemic in Wuhan, China: a modelling study," The Lancet Public Health, vol. 5, no. 5, pp. e261-e270, 2020.

[14] H. Solomon, D. Allen, S. Annan-Phan et al., "The effect of large-scale anticontagion policies on the covid-19 pandemic," Nature, vol. 1-9, 2020.

[15] G. Bonaccorsi, F. Pierri, M. Cinelli et al., "Economic and social consequences of human mobility restrictions under covid19," in Proceedings of the National Academy of Sciences, vol. 117, no. 27, pp. 15530-15535, 2020.

[16] W. Wang, Q.-H. Liu, J. Liang, Y. Hu, and T. Zhou, "Coevolution spreading in complex networks," Physics Reports, vol. 820, pp. 1-51, 2019.

[17] S. Funk, E. Gilad, C. Watkins, and V. A. A. Jansen, "The spread of awareness and its impact on epidemic outbreaks," Proceedings of the National Academy of Sciences, vol. 106, no. 16, pp. 6872-6877, 2009.

[18] J.-Q. Kan and H.-F. Zhang, "Effects of awareness diffusion and self-initiated awareness behavior on epidemic spreading - an approach based on multiplex networks," Communications in Nonlinear Science and Numerical Simulation, vol. 44, pp. 193-203, 2017.

[19] H. Wang, Li Qian, G. D’Agostino, S. Havlin, H. E. Stanley, and P. Van Mieghem, "Effect of the interconnected network structure on the epidemic threshold," Physical Review E, vol. 88, no. 2, Article ID 022801, 2013.

[20] M. J. Keeling, T. House, A. J. Cooper, and L. Pellis, "Systematic approximations to susceptible-infectious-susceptible dynamics on networks," PLoS Computational Biology, vol. 12, no. 12, Article ID e1005296, 2016.

[21] N. Antulov-Fantulin, A. Lančić, H. Štefančić, and M. Šikić, "FastSIR algorithm: a fast algorithm for the simulation of the epidemic spread in large networks by using the susceptibleinfected-recovered compartment model," Information Sciences, vol. 239, pp. 226-240, 2013.

[22] H.-F. Zhang and Z. Wang, "Suppressing epidemic spreading by imitating hub nodes' strategy," IEEE Transactions on Circuits and Systems II: Express Briefs, vol. 67, no. 10, pp. 1979-1983, 2019.
[23] H.-F. Zhang, J.-R. Xie, M. Tang, and Y.-C. Lai, "Suppression of epidemic spreading in complex networks by local information based behavioral responses," Chaos: An Interdisciplinary Journal of Nonlinear Science, vol. 24, no. 4, Article ID 043106, 2014.

[24] E. A. Fronhofer and F. Altermatt, "Classical metapopulation dynamics and eco-evolutionary feedbacks in dendritic networks," Ecography, vol. 40, no. 12, pp. 1455-1466, 2017.

[25] S. Venkatramanan, J. Chen, A. Fadikar et al., "Optimizing spatial allocation of seasonal influenza vaccine under temporal constraints," PLoS Computational Biology, vol. 15, no. 9, Article ID e1007111, 2019.

[26] T. Jessica, P. Nicola, Q. Zhang, Y. Moreno, and A. Vespignani, "Phase transitions in information spreading on structured populations," Nature Physics, vol. 16, no. 5, pp. 590-596, 2020.

[27] H.-F. Zhang, J.-R. Xie, H.-S. Chen, C. Liu, and M. Small, "Impact of asymptomatic infection on coupled disease-behavior dynamics in complex networks," EPL (Europhysics Letters), vol. 114, no. 3, p. 38004, 2016.

[28] J. Sun, L. Feng, J. Xie, Ma Xiao, D. Wang, and Y. Hu, "Revealing the predictability of intrinsic structure in complex networks," Nature Communications, vol. 11, no. 1, pp. 1-10, 2020.

[29] C. Xia, Z. Wang, C. Zheng et al., "A new coupled diseaseawareness spreading model with mass media on multiplex networks," Information Sciences, vol. 471, pp. 185-200, 2019.

[30] R. Pastor-Satorras, C. Castellano, P. Van Mieghem, and A. Vespignani, "Epidemic processes in complex networks," Reviews of Modern Physics, vol. 87, no. 3, pp. 925-979, 2015.

[31] C. Granell, S. Gomez, and A. Arenas, "Dynamical interplay between awareness and epidemic spreading in multiplex networks," Physical Review Letters, vol. 111, no. 12, Article ID 128701, 2013.

[32] E. Shim, A. Tariq, W. Choi, Y. Lee, and G. Chowell, "Transmission potential and severity of covid-19 in South Korea," International Journal of Infectious Diseases, vol. 93, pp. 339-344, 2020.

[33] K. Karako, P. Song, Y. Chen, and W. Tang, "Analysis of covid19 infection spread in Japan based on stochastic transition model," Bioscience Trends, vol. 14, no. 2, pp. 134-138, 2020.

[34] L. Rosenbaum, "Facing covid-19 in Italy - ethics, logistics, and therapeutics on the epidemic's front line," New England Journal of Medicine, vol. 382, no. 20, pp. 1873-1875, 2020.

[35] J. Yuan, M. Li, G. Lv, and Z. K. Lu, "Monitoring transmissibility and mortality of covid-19 in Europe," International Journal of Infectious Diseases, vol. 95, pp. 311-315, 2020.

[36] X. Marchand-Senécal, R. Kozak, S. Mubareka et al., "Diagnosis and management of first case of COVID-19 in Canada: lessons applied from SARS-CoV-1," Clinical Infectious Diseases, vol. 71, no. 16, pp. 2207-2210, 2020.

[37] M. Seyed, A. Shoukat, M. C. Fitzpatrick et al., "Projecting hospital utilization during the covid-19 outbreaks in the United States," in Proceedings of the National Academy of Sciences, vol. 117, no. 16, pp. 9122-9126, 2020.

[38] J. Rodriguez-Morales, V. Gallego, J. Escalera-Antezana et al., "Covid-19 in Latin America: the implications of the first confirmed case in Brazil," Travel Medicine and Infectious Disease, vol. 35, Article ID 101613, 2020. 